\title{
Subject Index Vol. 14, 1997
}

Acidosis 292 Action potentials 245 Acute cholecystitis 398

-, complications 192

pancreatitis 77

respiratory distress syndrome, esophageal resection 46

Adenocarcinoma 70 Advanced pancreatic cancer 113

surgical technology 450 Alveolar echinococcosis 229

hydatid disease 229 Anal canal 34

pressures 299 Anastomosis 88, 183 Angiotensin-converting enzyme

inhibitor 292 Anterior displaced anus 413 Appendicitis 195

Bacterial translocation 267,

284 Bacteriotherapy 284 Banga 469 Bariatric surgery 438 Benign biliary stricture 527

mesenchymal tumors 307 Bile duct fistula 272 Biliary injuries 534

obstruction 267

peritonitis, diagnosis 192

tract 423 Blood transfusion 2

Cancer prognosis 2 Capsectomy 241 Carcinoembryonic antigen

doubling time 393 Carcinoid 423

Carcinoma, gastric cardia 506 -, Vater's ampulla 319 Caroli's syndrome 123 Celiac axis infusion 113 c-erbB-2 252 c-erbB-3 252 Cholangiocellular carcinoma

381 Cholangiography 534 Cholecystectomy 192,429-, complications 534

Cholecystokinin 175 Chronic pancreatitis 107,148, 495

- $\quad$-, complications 540

Cirrhosis 344

Cisplatin 165

Clinical anal function 299 Clostridium difficile 222 Colon cancer 198, 444

- $\quad$-, splenic abscess 426

Colonoscopy 202

Colorectal cancer 311,393

Colovesical fistula(s) 56, 444

Computerized tomography

injury grading systems 277 Concurrent cancers 61 Constipation 413 Crile 469

Curative resection 123 Cushing 469 Cutaneous fistula 483 Cyclosporine A 136 Cytosolic free

$\mathrm{Ca} 2+92$

Diversion 222 Diverticular disease 56 DNA flow cytometry 61 Doppler ultrasound 344 Drainage 88, 119,241 Ductus cysticus 423 Dumping syndrome 512 Duodenal fistula, surgery 43

- $\quad$ injury 43

Duodenogastric reflux 175 Dysplasia 319

Early cancer 70 Endoscopic decompression

222 Enterocolitis 284 Enterocutaneous fistula 483 Epidermal growth factor 
receptor overexpression

252 Esophageal cancer 70, 88, 506 Esophagitis 175 Extended left hepatectomy 28 Extramucosal stricturoplasty

492

Fecal incontinence $409 \alpha$-Fetoprotein 557

Fistula 154

-, biliary 534

Foramen, Winslow hernia 119

Gallbladder 119

carcinoma, survival 169

perforation 192

surgery 272 Gallstone ileus 311 Gangrene 119 Gastrectomy 512 Gastric cancer 61,252,260,

$371,557,562$

- $\quad$ emptying 159

Gastrin 175

Gastrocolic omentum 315 Gastroduodenal ulcer, follow-up 366

Gastroesophageal reflux disease

235 Gastrohepatic omentum 315 Gastrointestinal bleeding 126

cancer 9

complications 136

hormone 512 Graft-vs.-host disease 23 Gram-negative bacteria causing

splenic abscess 426

Halsted 469

Helicobacter pylori, prevalence

366 Hemorrhoids, complications 52 Hepatectomy 92 Hepaticojejunostomy 527, 534

Hepatocellular carcinoma 165 ,

557 Hepatoid adenocarcinoma 557 Hernia repair 82

- $\quad$-, laparoscopic 433

Highly selective vagotomy 521

Hydatidosis 241

Hyperdynamic circulation 344

Hypoproteinemia 562

Iatrogenic bile duct injuries

527 Immunosuppression 136 Incontinence 34 Infected pancreatic necrosis 77 Inferior vena cava abnormality

65

Inguinal hernia 361,433 Inner ring closure 82 Internal hernia 119,315 Intestinal obstruction

315,492

- $\quad$ transplantation 23

IntracellularcAMP 92

Intraesophageal pressure 245

Intrahepatic hematoma 387

Intubation 154

Ischemia 205 Isolated hepatocyte 92

Kaposi sarcoma 136 Kocher 469 K-ras 552

Lactate 292 
Lactobacillus acidoph ilus 284

plantarum 284

rhamnosus 284 Lactulose 267 Laparoscopic anti-reflux

surgery 235

cholecystectomy $169,398,450$

colon resection 444

gastric banding 438

Nissen fundoplication 235

sigmoidectomy 56

surgery 450

wedge resection, stomach 562

Laparoscopy 39, 82, 272, 429,

433 Lesser sac exploration 119 Linitis plastica 419 Lipiodol 165 Liver 241

blood flow 344

transplantation 229, 344, 387

tumors 198 Local anesthesia 361

excision, rectal cancer 304 Low anterior resection 299 Lymphadenectomy 371

Malignant melanoma, esophagus 552 Malnutrition 512 Mayo 469 Mesenteric vessels 205

\section{KAHGER}

E-Mail karger@,karger.ch Fax+ 41613061234 http:// www. karger. ch

(C) 1997 S. KargerAG, Basel

575

Metachronous hepatic metastasis 393

Methotrexate 284

Microcirculation 292

Microflora 284

Mirizzi syndrome 272

MitomycinC 165

Moore procedure 409

Morbid obesity 438

Multidirectional invasion, carcinoma 319

Multiple rubber band ligation 52

Neoplasms 154 Neoprene 187 Nephrotic syndrome 562 Nonocclusive mesenteric infarct 292 Northern blot analysis 252 Nutrition 260

Occult gastrointestinal blood

loss, source 126 Octreotide 483 Open packing 77 Outlet obstruction, surgery 413 p53 552

Pacesetter potentials 245

Pain 148

Palliation 9

Pancreas 148, 183

Pancreatic cancer 159

duct drainage 495

fíbrosis 101 
fistula 187,546

growth 175

head, local resection 148

hemorrhage 107

resection 101, 187

schwannoma 307

stump 187

surgery, complications 159,187

tumors 101

Pancreaticoduodenectomy 546 Pancreaticojejunostomy 495,

546 -, longitudinal 148 Parasitic liver disease 229 Parenteral nutrition 483 Percutaneous liver

biopsy 387 Perforated peptic ulcer 521 Periampullary cancer 159 Peritonitis 210 Phlegmonous

gastritis 210 Pneumoperitoneum 202, 429 Pneumoretroperitoneum 202 Portal aneurysm 65

hypertension 344

systemic shunt 344

vein abnormalities 65 Postoperative small-bowel

obstruction 311

- $\quad$ infectious complications 2

Postprandial symptoms 260

Pouch reconstruction 260

Pregnancy, colon cancer 198

Prevalence, appendicitis 195

Prolamin 187

Prostaglandin I2 92

Pseudocysts 540

Pseudomembranous colitis 222

Puborectalis muscle 34 Pylorus-preserving Whipple procedure 159

Radiation enteritis 492 Rectal cancer 304

neoplasm 299

prolapse 34, 409 Rectopexy 409 Reflux esophagitis 512 Renal amyloidosis 562

transplantation 136 Repeated transcatheter arterial

embolization 165

Scirrhous carcinoma 419 Segmental bilioenteric bypass

169 Senn 469 Serosal patch 43 Severe obesity 355

- $\quad$-, operative choice 355

Shouldice repair 361

Single rubber band ligation 52 Single-strand conformation

polymorphism, 'cold' 552 Slow waves 245 Small-cell cancer 61 Splenic abscess 426

- $\quad$ injury 277

Split gastrojejunostomy 175 Squamous cell carcinoma 70 Stab wound 39 Stent 154

Stomach cancer 419 Strangulation 119 Strictures, biliary 534

Subcutaneous emphysema 202 Subtotal colectomy 222 Superficial invasion, carcinoma

319 Suprapubic catheterisation 404 Synchronous hepatic metastasis

393 
$\gamma \delta$ T cell receptor 23 Terminal ileum 119 Thoracotomy 154 Thromboangiitis obliterans 205 Thrombosis 205 Thromboxane A2 92 Thyroid 469 Total gastrectomy 260 Tracheoesophageal fistula 154 Transomental herniation 315 Transplantation immunology

23 Transsphincteric surgery 304

Unilobar Caroli's syndrome

123 Urethral catheterisation 404

Vancomycin 222 Vertical-banded gastroplasty

355

Whipple, partial pancreato-

duodenectomy 159 - procedure 187,546 Wirsung duct occlusion 187

576

DigSurgVol. 14,1997

Subject Index 